\title{
Influence Factor on Magnetization Property of Carbonyl Iron-based Magnetorheological Fluids
}

\author{
Daoming Wang*, Bin Zi, Sen Qian, Jun Qian, and Yishan Zeng \\ School of Mechanical Engineering, Hefei University of Technology, Hefei 230009, China
}

(Received 7 August 2016, Received in final form 3 November 2016, Accepted 14 November 2016)

\begin{abstract}
Magnetization property is a critical factor for magnetorheological fluids (MRFs) to achieve the liquid-solid transition. The main focus of this study is on exploring the influence factors on magnetization properties of MRFs. In this paper, a theoretical analysis is performed to discuss the magnetization characteristics of MRFs firstly. Then, a method for the preparation of carbonyl iron-based MRFs is illustrated and five MRFs samples with various material parameters are prepared. It is succeeded by a series of experiments on testing the hysteresis loop and the magnetization curve of each sample and the influence factors are compared and analyzed. Experimental results indicate that there is basically no hysteresis phenomenon on MRFs which exhibits superparamagnetic behavior at room temperature. A surfactant coating on magnetic particles can slightly improve the MRFs magnetization. Additionally, the magnetic susceptibility and the saturation magnetization both increase with the particle concentration, whereas the influence of particle diameter is relatively very small. Moreover, as the temperature increases, the magnetization decreases and the declining rate accelerates gradually.
\end{abstract}

Keywords : magnetorheological fluids, magnetization property, surfactant coating, particle diameter, particle concentration, temperature effect

\section{Introduction}

Magnetorheological fluids (MRFs) are suspensions prepared by dispersing micron-sized magnetic particles uniformly into a carrier liquid [1-6]. Meanwhile, a variety of proprietary additives is commonly added to discourage particle sedimentation, improve lubricity and inhibit wear. MRFs cleverly combines the strong magnetism of solid particles and the good fluidity of the carrier liquid together, thus possessing excellent liquid-solid phase transition characteristics in response to a magnetic field [7-9]. Moreover, the transition process is characterized by an instant and reversible response, easy and precise control, as well as low-energy consumption [10-12]. As a consequence, MRFs have been widely used in many energy absorption and precise control applications, such as vibration dampers, shock absorbers, servo valves, clutches, brakes and polishing devices [13-21].

Material properties of MRFs are critical to the working

(C)The Korean Magnetics Society. All rights reserved.

*Corresponding author: Tel: +86-551-62901606

Fax: +86-551-62901606, e-mail: cumtcmeewdm@hotmail.com stability, control accuracy and even service life of MRFbased devices [4]. Therefore, considerable research works related to this issue have been carried out. For instance, Bica [22] focused on exploring the effect of temperature and magnetic field on the electrical conductivity of MRFs. Tian et al. [23] performed an experimental study on the impact of material, roughness and surface morphology of the disk on power transmission properties of MRFs. Gómez-Ramírez et al. [24] studied on the wall slip characteristics of MRFs using the experimental method. Wiehe et al. [25] measured the influence factors on the durability of MRFs through lots of experiments. Yildirim and Genc [26] investigated the thermal conductivity of MRFs in three temperature intervals. Routhier and StAmant [27], Sarkar and Hirani [28] successively researched on the squeeze enhancement effect on the shear stress of MRFs. Liu et al. [29] experimentally studied on the effect of additional nanometer $\mathrm{Fe}_{3} \mathrm{O}_{4}$ on the sedimentation stability, zero field viscosity, and shear yield stress of silicone oil-based MRFs. Wang et al. [30] measured the viscosity and the shear stress of MRFs using a selfdeveloped parallel disk shear stress testing device. Yao et al. [31] researched the delay time of MRFs in porous 
foam metal.

It is well known that the chain formation of MRFs is based on the polarization and interaction of magnetic particles under the magnetic field effect. Therefore, the magnetization property can be regarded as a critical factor for MRFs to achieve the liquid-solid phase transition. However, there are rarely few works regarding to the magnetization mechanism and properties of MRFs as reported above. Therefore, a thorough study on magnetization properties of MRFs is necessary which can help ameliorate the preparation technology so as to improve the material properties of MRFs.

Consequently, the technical novelty of this study is to reveal the influence factors on magnetization properties of MRFs by mean of comprehensive theoretical analysis and experimental investigation. In this paper, five kinds of carbonyl iron-based MRFs samples are prepared using a proposed base fluid replacement method. Then, theoretical analysis and experimental investigation are performed successively for the systematic study of influence factors on magnetization characteristics of MRFs. Experimental results concerning the influences of surfactant coating, particle size and concentration, as well as temperature on the magnetization of MRFs samples are presented and discussed. Research findings in this study can provide a theoretical and experimental guidance for the preparation of high performance MRFs.

\section{Theoretical Analysis}

Magnetic particles in MRFs are typically ferromagnetic or ferrimagnetic mediums. As a population of non-interacting particles, ferromagnetic properties of a small amount of suspended particles in MRFs are negligible. Thus, magnetization rules of MRFs under an external field can be described by the Langevin function [32]

$$
M_{\mathrm{f}}=n m\left(\operatorname{ctg} \xi-\frac{1}{\xi}\right)
$$

where $M_{\mathrm{f}}$ is the magnetization of MRFs, $n$ is the particle number per unit volume, $m$ is the magnetic moment of particles, $\xi$ is the Langevin argument.

When the applied magnetic field is weak, i.e. $\xi \ll 1$, the magnetization increases linearly with the Langevin argument. Then

$$
M_{\mathrm{f}}=n m \xi=\left(\frac{\mu_{0} n m}{k_{0} T}\right) H=\chi_{\mathrm{f}} H
$$

where $\mu_{0}$ is the vacuum permeability, $T$ is the thermodynamic temperature, $k_{0}$ is the Boltzmann constant, $k_{0} \approx 1.38 \times 10^{-23} \mathrm{~J} / \mathrm{K}, H$ is the magnetic field intensity, $\chi_{\mathrm{f}}$ is the magnetic susptibility of MRFs, $\chi_{\mathrm{f}}=\mu_{0} n m / k_{0} T$.

With the increase of the magnetic field, the magnetization gradually tends to a stable value. At this time, magnetic moments of particles all orient along the field direction. Then, the magnetization of MRFs is expressed as

$$
M_{\mathrm{f}}=M_{\mathrm{fs}}\left(1-\frac{k_{0} T}{\mu_{0} m H}\right)=n m\left(1-\frac{k_{0} T}{\mu_{0} m H}\right)
$$

where $M_{\mathrm{fs}}$ is the saturation magnetization of MRFs.

As the temperature increases, the random thermal motion of particles enhances correspondingly. This feature can be described by the Langevin model as

$$
\xi=\frac{\mu_{0} m H}{k_{0} T}
$$

Similarly, a volume expansion occurs to MRFs with increasing temperature. It results in a decrease of the particle concentration, thus reduceing the magnetic susceptibility of MRFs. The relationship between $n$ and $T$ is given by

$$
n=\alpha_{\mathrm{f}} \frac{\Delta n}{\Delta T}
$$

where $\alpha_{\mathrm{f}}$ is the thermal expansion coefficient of MRFs.

The increase of temperature also destroys the ordered arrangement of magnetic moments of particles. In specific, when the temperature reaches close to the Curie temperature of the particulate material, the magnetic moment declines in proportion to $\sqrt{T-T_{\text {cr }}}$ as the temperature increases. However, once it exceeds the Curie temperature, the ordered arrangement of magnetic moment is destroyed that makes the particles transform from ferromagnetic state to superparamagnetic state. In a broad temperature range, the magnetic moment and the temperature follows the relationship as

$$
m=m_{0} \sqrt{\frac{3\left(T_{\mathrm{cr}}-T\right)}{T_{\mathrm{cr}}}}
$$

where $m_{0}$ is the magnetic moment of particles at absolute zero $(T=0 \mathrm{~K})$ and $T_{\text {cr }}$ is the Curie temperature of the particulate material.

where $m_{0}$ is the magnetic moment of particles at absolute zero $(T=0 \mathrm{~K})$ and $T_{\text {cr }}$ is the Curie temperature of the particulate material.

Referring to Eq. (1), the magnetization of MRFs is mainly determined by three parameters: the Langevin argument $\xi$, the magnetic moment of particles $m$ and the particle number per unit volume $n$. As described in Eqs. (4) to (6), the above-mentioned three parameters are all 
affected by the temperature. Thus, the temperature dependence of MRFs magnetization under a given magnetic field $H$ is mainly determined by the temperature effect on $\xi, m, n$ and it can be given as

$$
\begin{aligned}
\left(\frac{\partial M_{\mathrm{f}}}{\partial T}\right)_{H} & =\left(\frac{\partial M_{\mathrm{f}}}{\partial T}\right)_{H, n, m}+\left(\frac{\partial M_{\mathrm{f}}}{\partial m}\right)_{H, n, T}\left(\frac{\partial m}{\partial T}\right)_{n} \\
& +\left(\frac{\partial M_{\mathrm{f}}}{\partial n}\right)_{H, T, m}\left(\frac{\partial n}{\partial T}\right)_{m}
\end{aligned}
$$

It is found through theoretical analysis that magnetization properties of MRFs, including magnetic susceptibility and saturation magnetization, are all influenced by the magnetic moment and concentration of particles, as well as the temperature. Consequently, an experimental investigation is required for a further probe into the influence factors on magnetization properties of MRFs.

\section{Experiments}

\subsection{MRFs sample preparation}

The preparation of high performance MRFs is of significant importance to the development of MRF-based devices. In this paper, we propose a base fluid replacement method for the MRFs sample preparation. The process is shown in Fig. 1 and it is summarized as follows:

- Several weighed magnetic particles are mixed together with a suitable amount of surfactant and absolute ethyl alcohol in a stainless steel container.

- High-speed stirring and ultrasonic decomposition are successively carried out on the mixture via a motordriven stirrer and an ultrasonic disperser for an intensive mixing. The JJ-1 stirrer is provided by Shanghai Kesheng Instrument Co., Ltd and the ultrasonic disperser (model VCY-900s) is from Shanghai Yanyong Ultrasonic Instrument Co., Ltd. The stirring speed is $500 \mathrm{r} / \mathrm{min}$ and the stirring time is 2 hours.

- The mixture is placed in a DZF-6021 vacuum drying oven (VDO) from Shanghai Zhongyou Instrument Co., Ltd with a constant temperature of $80^{\circ} \mathrm{C}$ for drying out the absolute ethyl alcohol. It is followed by a grinding operation on the dried mixture with a ball grinder (BM6, Beijing Grinder Instrument Equipment Co., Ltd) for 8 hours, and then the modified particles are obtained.

- The modified particles are mixed with the carrier liquid and several additional additives in a certain proportion. Then, the MRFs sample is acquired after once more high-speed \& constant temperature stirring on the mixture in a HH-2 constant temperature bath from Changzhou Jingda Instrument Manufacturing Co., Ltd. The stirring speed is $500 \mathrm{r} / \mathrm{min}$, the temperature is $30^{\circ} \mathrm{C}$ and the stirring lasts for 2 hours.

Material selections for the MRFs preparation are given as follows: the surfactant-coated carbonyl iron particles (except sample M5\#) with average diameters of $2 \mu \mathrm{m}$ and $7 \mu \mathrm{m}$ are used as magnetic particles; the methylsilicone oil with a kinematic viscosity of $100 \mathrm{~mm}^{2} / \mathrm{s}$ is used as the carrier liquid; the silane coupling agent, bentonite and graphite particles are used as additives. Referring to the preparation process in Fig. 1, five kinds of MRFs samples with different material parameters are prepared and specific parameters of each sample are given in Table 1.

Table 1. Specific parameters of MRFs samples.

\begin{tabular}{lccccc}
\hline \hline Sample No. & M1\# & M2\# & M3\# & M4\# & M5\# \\
\hline Particle diameter $(\mu \mathrm{m})$ & 2 & 7 & 7 & 7 & 7 \\
$\begin{array}{l}\text { Carrier liquid viscosity } \\
\left(\mathrm{mm}^{2} / \mathrm{s}\right)\end{array}$ & 100 & 100 & 100 & 100 & 100 \\
$\begin{array}{l}\text { Particle mass fraction } \\
(\%)\end{array}$ & 66.9 & 33.9 & 47.3 & 66.9 & 66.9 \\
\hline Surfactant-coated? & \multicolumn{5}{c}{ Yes } \\
\hline
\end{tabular}



Fig. 1. (Color online) Preparation process of MRFs simples. 
Table 2. Main parameters of JDAW-2000D VSM.

\begin{tabular}{cc}
\hline \hline Technical parameters & Value/Range \\
\hline Magnetic moment & $10^{-3} \sim 300 \mathrm{emu}$ \\
Control temperature & $-125 \sim 773 \mathrm{~K}$ \\
Magnetic field intensity & $0 \sim 1280 \mathrm{kA} / \mathrm{m}$ \\
Temperature precision & $\pm 1.5 \mathrm{~K}$ \\
Measurement precision & $\leq 1 \%$ \\
\hline
\end{tabular}

To analyze the influence of material parameters on MRFs performances, the following matches are utilized: M1\# and M4\# are compared to analyze the effect of particle diameter $(2 \mu \mathrm{m}$ and $7 \mu \mathrm{m})$; M2\#, M3\# and M4\# are compared to analyze the effect of particle mass fraction (33.9\%, $47.3 \%$ and $66.9 \%$ ); M4\# and M5\# are compared to analyze the effect of surfactant coating (uncoated and coated).

\subsection{Experimental apparatus and contents}

Generally, magnetization properties of a sample material can be measured by vibrating sample magnetometer (VSM), Gouy magnetic balance or $\mathrm{BH}$ meter. In this study, a JDAW-2000D VSM from Changchun INPRO Magnetoelectric Technology Co., Ltd is used for the magnetization measurement of MRFs samples. A photograph of the VSM is shown in Fig. 2 and Main parameters are given in Table 2.

The experiments are carried out to measure the magnetization characteristics of MRFs samples with various material parameters. Main experimental contents include: i) the hysteresis loop of MRFs at room temperature (RT); ii) the effect of surfactant coating on magnetization properties of MRFs; iii) magnetization curves of MRFs with different particle diameters and mass fractions; iv) variations of MRFs magnetization with temperature.

\section{Results and Discussion}

Figure 3 and Fig. 4 separately show the hysteresis loop

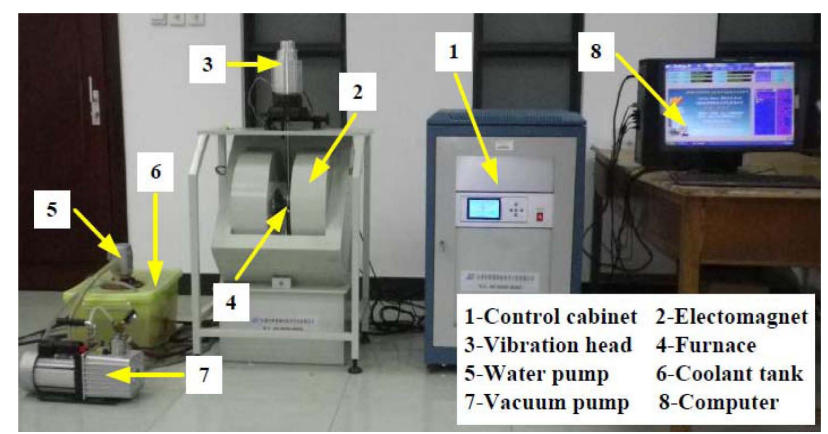

Fig. 2. (Color online) Photograph of JDAW-2000D VSM.

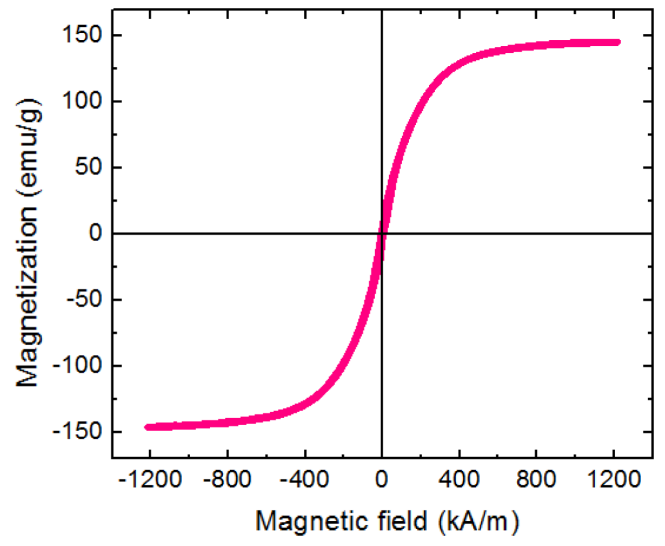

Fig. 3. (Color online) Hysteresis loop of M4\# at RT.

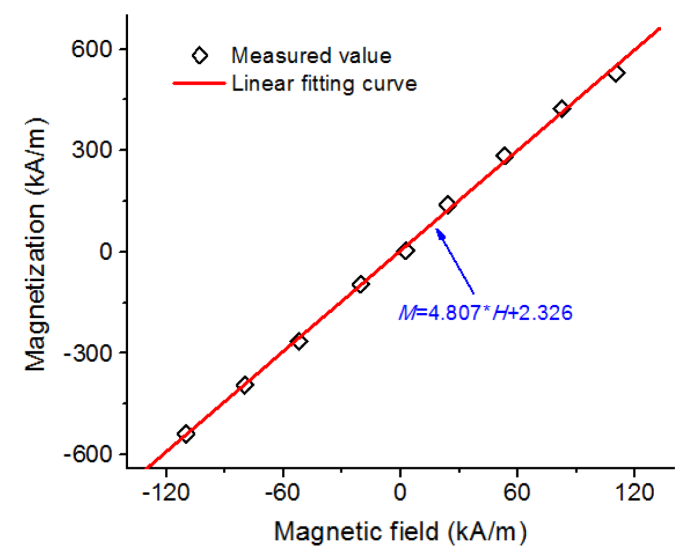

Fig. 4. (Color online) Variation of magnetization with magnetic field in low field range.

of M4\# at RT and the variation of magnetization with magnetic field in the low field range. As plotted in the two figures, the MRFs possess a strong magnetism at RT. Moreover, unlike ordinary ferromagnetic materials, there is basically no hysteresis phenomenon on MRFs which exhibit superparamagnetic behavior during the whole magnetization process. In the low field range less than $130 \mathrm{kA} / \mathrm{m}$, the magnetization increases proportionally with the magnetic field and the magnetic susceptibility is obtained as $\chi=4.807$ using the linear fitting method. However, the magnetic susceptibility gradually decreases with increasing magnetic field in the high field range. Moreover, the increasing trend of the magnetization slows down gradually till it reaches the saturation state. From then on, the magnetization remains basically unchanged with a value of about $145.2 \mathrm{emu} / \mathrm{g}$.

In this study, the silane coupling agent $\mathrm{KH}-550$ is used as the surfactant. The microstructures of uncoated particles and coated particles are characterized using a scanning electron microscope (SEM) and the SEM images of the 


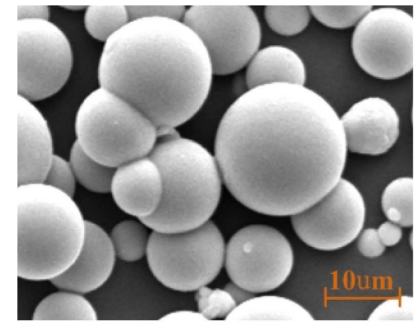

(a) uncoated

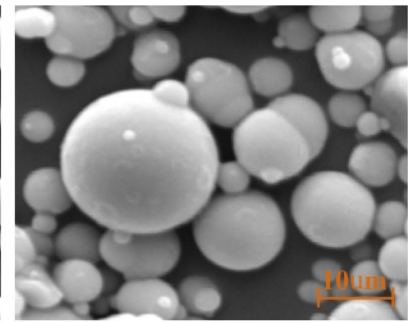

(b) coated
Fig. 5. (Color online) SEM images of particles before \& after surfactant coating.

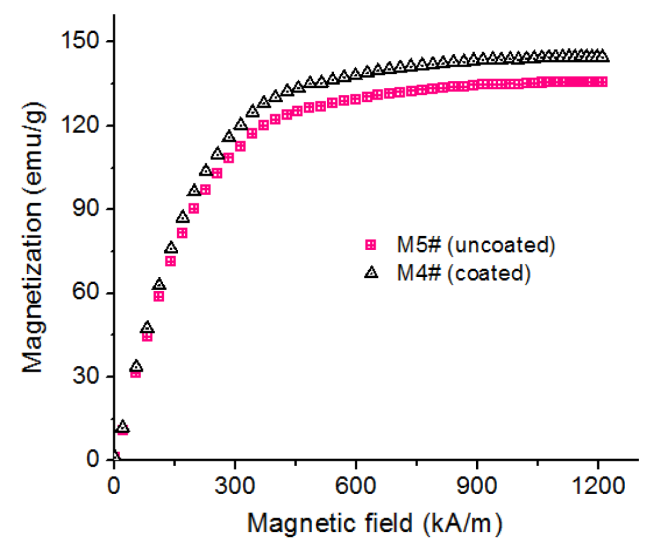

Fig. 6. (Color online) Magnetization curves of MRFs samples before \& after surfactant coating.

particles with an average diameter of $7 \mu \mathrm{m}$ are obtained as plotted in Fig. 5. It can be seen in Fig. 5 that the coating effect is good and there is no significant defect on the particles, which in return proves the rationality of the surfactant coating process.

To reveal the effect of surfactant coating on magnetization properties of MRFs, two samples namely M4\# and M5\# are selected for the measurements. Magnetization curves of the two samples are shown in Fig. 6.

In Fig. 6, the saturation magnetizations of MRFs samples before $\&$ after surfactant coating are about $135.9 \mathrm{emu} / \mathrm{g}$ and $145.2 \mathrm{emu} / \mathrm{g}$, respectively. According to the measured hysteresis loop of $7 \mu \mathrm{m}$-sized carbonyl iron particle in Literature [4], the saturation magnetization of magnetic particles at RT is nearly $204.2 \mathrm{emu} / \mathrm{g}$. Obviously, the saturation magnetization of MRFs samples after surfactant coating is slightly higher than the product of the particle saturation magnetization and the particle mass fraction (that is, $204.2 \mathrm{emu} / \mathrm{g} \times 66.9 \%=136.6 \mathrm{emu} / \mathrm{g}$ ). The main reason for the phenomenon is due to the fact that the surfactant coating effect can improve the polarization capability of magnetic particles, which thus enhances the magnetization properties of MRFs.

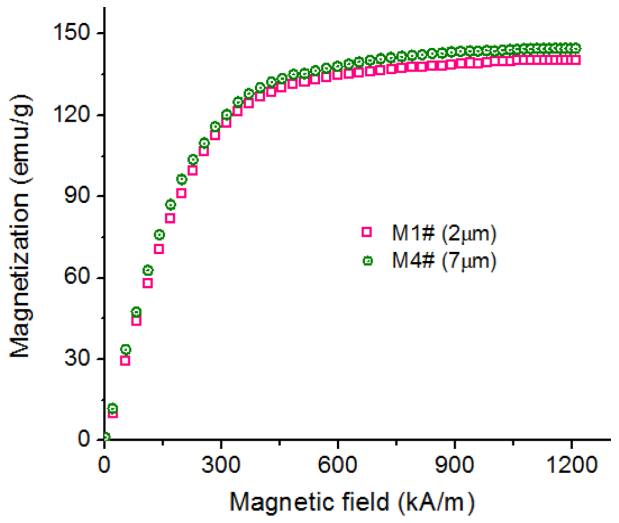

Fig. 7. (Color online) Magnetization curves of MRFs samples with. different particle diameters.

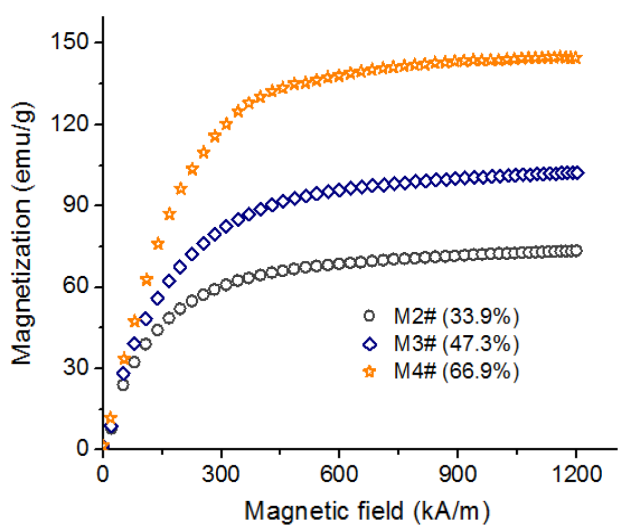

Fig. 8. (Color online) Magnetization curves of MRFs samples with different particle mass fractions.

As discussed in Section 2, magnetization properties of MRFs are influenced by the magnetic moment and the concentration of particles. The following tests are conducted to explore the influence laws of particle diameter and mass fraction on the magnetization of MRFs. In the experiments, magnetization curves of four samples, namely M1\#, M2\#, M3\# and M4\#, are tested and compared as shown in Fig. 7 and Fig. 8.

It is obvious that the magnetization processes are basically similar among MRFs samples with different parameters. At the beginning, the magnetization increases rapidly with magnetic field, and then, the increasing trend slows down gradually till the MRFs saturate. Moreover, there exists a certain difference among the values of magnetizations for different samples, which embodies in the increase of magnetic susceptibility and saturation magnetization with the particle mass fraction. For the samples with different particle mass fractions of $33.9 \%$, $47.3 \%$ and $66.9 \%$, the saturation magnetizations are $73.4,102.2$ and $145.2 \mathrm{emu} / \mathrm{g}$ respectively. Moreover, the 


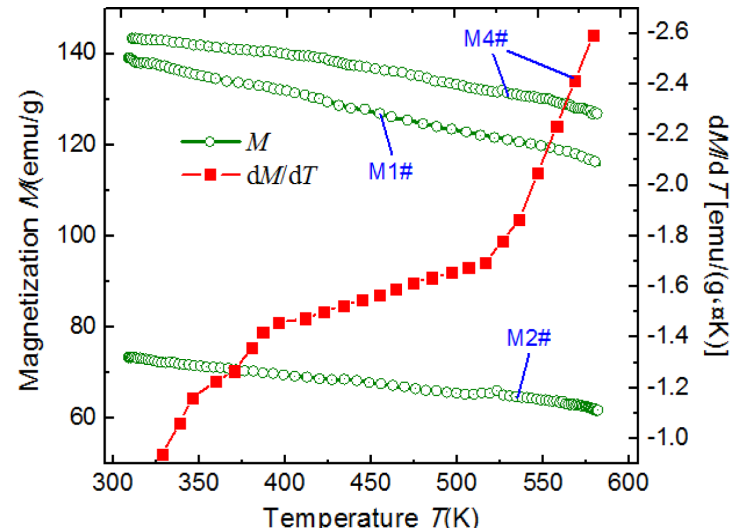

Fig. 9. (Color online) Variations of magnetization with temperature for MRFs samples.

effect of particle diameter on the magnetization is relatively very small, as depicted in Fig. 7. In specific, the saturation magnetization of M4\# ( $7 \mu \mathrm{m}$-sized) is $4.2 \mathrm{emu} /$ $\mathrm{g}$ larger than that of M1\# (2 $\mu \mathrm{m}$-sized) for a same particle mass fraction of $66.9 \%$. The phenomenon can be explained by the fact that the ratio of regularly arranged atomic structures increases with the particle size which therefore leads to an increase of the magnetic moment for per unit mass particles.

As theoretically analyzed in Section 2, the temperature can simultaneously influence the Brown motion, magnetic moment and mass fraction of particles, thus affecting magnetization properties of MRFs. Variations of magnetization with temperature for three samples namely M1\#, M2\# and M4\# are measured as presented in Fig. 9. In the experiments, the magnetic field is $1200 \mathrm{kA} / \mathrm{m}$ and the temperature increases from $310 \mathrm{~K}$ to $580 \mathrm{~K}$ in increments of $10 \mathrm{~K}$.

In Fig. 9, the variation laws of magnetization with temperature are basically the same among different samples. As the temperature increases, a certain degree of degradation occurs to the magnetization characteristics of MRFs. Under a same magnetic field, the magnetization decreases gradually with the increase of temperature and the decreasing rate becomes faster at a higher temperature. Taking sample M4\# for example, its magnetization decreases from 144.3 to $126.5 \mathrm{emu} / \mathrm{g}$ as the temperature rises from 310 to $580 \mathrm{~K}$, a decline ratio of about $12.3 \%$. This phenomenon is possibly induced by the degradation of particle polarization ability and the destruction of surfactant coating effect on particles at high temperatures.

\section{Conclusions}

This study is concerned with the influence factors on magnetization properties of carbonyl iron-based magnetorheological fluids by means of both theoretical analysis and experimental research. The effects of surfactant coating, particle diameter, particle mass fraction and temperature on the magnetization of MRFs are measured and discussed. They reveal that the MRFs exhibit superparamagnetic behavior in the magnetization process and the magnetic susceptibility remains basically unchanged with a value of $\chi=4.807$ in the low field range less than $130 \mathrm{kA} / \mathrm{m}$. Moreover, both the magnetic susceptibility and the saturation magnetization of MRFs increase with the particle mass fraction whereas the effect of particle diameter is proved to be very small. The magnetization of MRFs can be improved slightly by a surfactant coating on magnetic particles. Furthermore, the magnetization of MRFs decreases as the temperature increases and the decreasing rate accelerates gradually with increasing temperature.

\section{Acknowledgment}

The authors gratefully acknowledge the National Natural Science Foundation of China (51505114), the Anhui Provincial Natural Science Foundation (1608085QE116), the China Postdoctoral Science Foundation Funded Project (2016T90561) and the Fundamental Research Funds for the Central Universities (JZ2016HGTB0717) for the financial support in this work.

\section{References}

[1] Y. H. Huang, Y. H. Jiang, X. B. Yang, and R. Z. Xu, J. Magn. 20, 317 (2015).

[2] D. Wang and Y. Hou, J. Intel. Mat. Syst. Str., 24, 640 (2013).

[3] S. G. Sherman and N. M. Wereley, J. Appl. Phys. 115, 17 (2014).

[4] D. Wang, B. Zi, Y. Zeng, Y. Hou, and Q. Meng, J. Mater. Sci. 49, 24 (2014).

[5] S. Rahimi and D. Weihs, J. Magn. 21, 2 (2016).

[6] M. Ashtiani, S. H. Hashemabadi, and A. Ghaffari, J. Magn. Magn. Mater. 374, (2015).

[7] D. M. Wang, Y. F. Hou, and Z. Z. Tian, Smart Mater. Struct. 22, 2 (2013).

[8] X. Wu, X. Xiao, Z. Tian, F. Chen, and J. Wang, J. Magn. 21, 2 (2016)

[9] J. de Vicente, D. J. Klingenberg, and R. Hidalgo-Alvarez, Soft Matter. 7, 8 (2011).

[10] K. P. Hong, Y. K. Cho, B. C. Shin, M. W. Cho, S. B. Choi, S. W. Cho, and J. J. Jae, Mater. Manuf. Process 27, 10 (2012).

[11] H. H. Sim, S. H. Kwon, and H. J. Choi, Colloid. Polym. 
Sci. 291, 4 (2013).

[12] S. Y. Kim, S. H. Kwon, Y. D. Liu, J. S. Lee, C. Y. You, and H. J. Choi, J. Mater. Sci. 49, 3 (2014).

[13] G. McLaughlin, W. Hu, and N. M. Wereley, J. Appl. Phys. 115, 17 (2014).

[14] A. Milecki and M. Hauke, Mech. Syst. Signal. Pr. 28, (2012).

[15] X. B. Yang, Y. H. Jiang, Y. H. Huang, R. Z. Xu, H. G. Piao, G. M. Jia, and X. Y. Tan, J. Magn. 19, 345 (2014).

[16] A. S. Shafer and M. R. Kermani, IEEE-ASME T. Mech. 16, 6 (2011).

[17] D. Wang, B. Zi, Y. Zeng, F. Xie, and Y. Hou, Smart Mater. Struct. 24, 5 (2015).

[18] D. Wang, Y. Hou, Q. Meng, and Z. Tian, Smart Mater. Struct., 22, 8 (2013).

[19] O. Erol, B. Gonenc, D. Senkal, S. Alkan, and H. Gurocak, J. Intell. Mater. Syst. Struct. 23, 4 (2012).

[20] A. K. Singh, S. Jha, and P. M. Pandey, Mater. Manuf. Process 27, 4 (2012).

[21] M. Niranjan, S. Jha, and R. K. Kotnala, Mater. Manuf. Process 29, 4 (2014).
[22] I. Bica, Phys. Rev. B 371, 1 (2006).

[23] Z. Tian, Y. Hou, and N. Wang, J. Funct. Mater. 42, 11 (2011).

[24] A. Gómez-Ramírez, M. T. López-López, F. GonzálezCaballero, and J. D. G. Durán, Rheol. Acta. 51, 9 (2012).

[25] A. Wiehe, C. Kieburg, and J. Maas, J. Phys.: Conference Series 412, 1 (2013).

[26] G. Yildirim and S. Genc, Smart Mater. Struct. 22, 8 (2013).

[27] G. Routhier and Y. St-Amant, International Workshop on Smart Materials, Structures \& NDT in Aerospace, Montreal, Canada, 2-4 November (2011).

[28] C. Sarkar and H. Hirani, Smart Mater. Struct. 22, 11 (2013).

[29] X. Liu, L. Wang, H. Lu, Q. Chen, and Z. Wang, Mater. Manuf. Process 30, 2 (2015).

[30] D. Wang, B. Zi, Y. Zeng, F. Xie, and Y. Hou, P. I. Mech. Eng. C-J Mec., doi: 10.1177/0954406215621099 (2015).

[31] X. Yao, Z. Chen, H. Qin, Q. Yu, and C. Li, Optoelectron. Adv. Mat. 10, 3-4 (2016).

[32] M. M. Maiorov and A. Cebers, Magnetohydrodynamics 19, 4 (1983). 